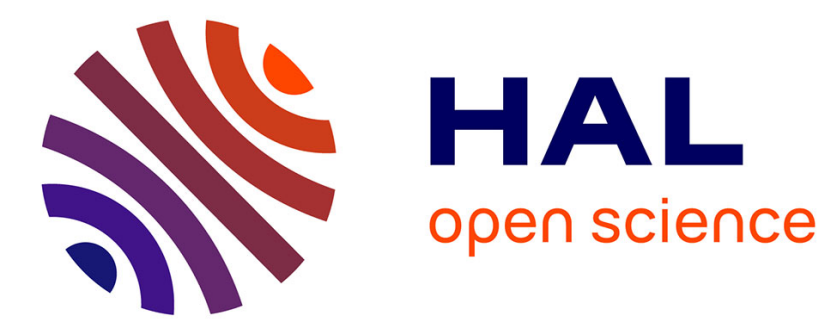

\title{
On human models for collaborative robots
}

Rachid Alami

\section{To cite this version:}

Rachid Alami. On human models for collaborative robots. 2013 International Conference on Collaboration Technologies and Systems (CTS, May 2013, San Diego, United States. hal-01053642

\section{HAL Id: hal-01053642 \\ https://hal.science/hal-01053642}

Submitted on 31 Jul 2014

HAL is a multi-disciplinary open access archive for the deposit and dissemination of scientific research documents, whether they are published or not. The documents may come from teaching and research institutions in France or abroad, or from public or private research centers.
L'archive ouverte pluridisciplinaire HAL, est destinée au dépôt et à la diffusion de documents scientifiques de niveau recherche, publiés ou non, émanant des établissements d'enseignement et de recherche français ou étrangers, des laboratoires publics ou privés. 


\title{
On Human Models for Collaborative Robots
}

\author{
Rachid Alami \\ CNRS, LAAS \\ Univ de Toulouse \\ 7 avenue du colonel Roche,F-31400 Toulouse, France \\ Rachid.Alami@laas.fr
}

INVITED PAPER

\author{
Keywords-Human-robot collaboration, Affordances, Human- \\ aware task planning
}

\section{INTRODUCTION}

Human-robot collaboration requires to equip the robot with explicit reasoning on the human and on its own capacities to achieve its tasks in a collaborative way with a human partner.

We present a robot control system which has been especially designed for a cognitive robot which shares space and task with a human. We have adopted a constructive approach based on effective individual and collaborative skills. The system is comprehensive since it aims at dealing with a complete set of abilities articulated so that the robot controller is effectively able to conduct a collaborative task with a human partner in a flexible manner

These abilities include geometric reasoning and situation assessment based essentially on perspective-taking and affordances, management and exploitation by the robot of each agent beliefs (human and robot) in a separate cognitive model, human-aware task planning and human and robot interleaved plan achievement

\section{A DECISIONAL FRAMEWORK}

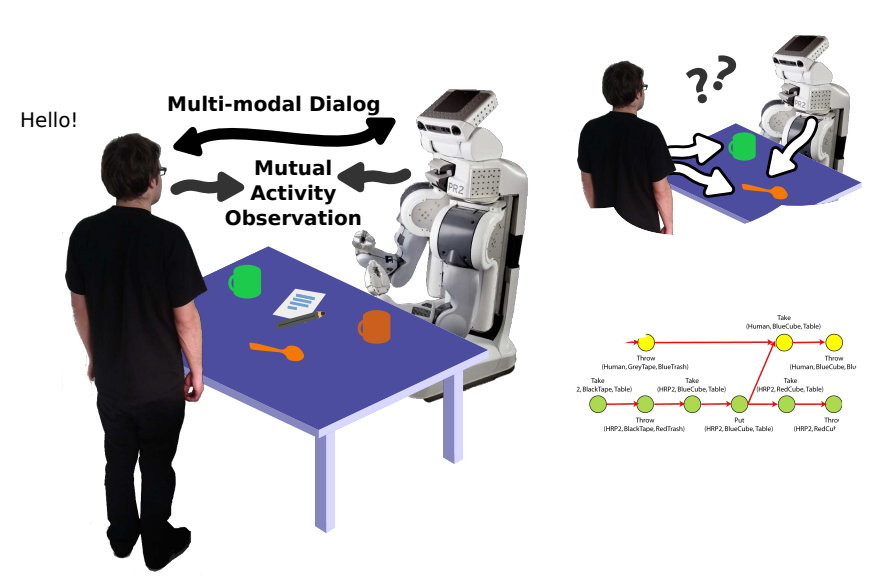

Figure 1. Robot reasoning about HRI and anticipation of human activities: sources of information are multi-modal dialogue, and observation of environment and human activity
We envision HRI in a context where two agents (a human and a robot) share a common space and exchange information through various modalities. Our aim is to endow the robot with an explicit consideration of the human and with the ability to manage its interactions with him (Figure 1). This must be considered at the architecture level as well as at the task/motion planning and execution level.

We have devised a decisional framework for human-robot interactive task achievement that is aimed to allow the robot not only to accomplish its tasks but also to produce behaviors that support its engagement vis-a-vis its human partner and to interpret human behaviors and intentions. Together and in coherence with this framework, we have developed and experimented various task planners and interaction schemes that allow the robot to select and perform its tasks while taking into account explicitly the human abilities as well as the constraints imposed by the presence of humans, their needs and preferences.

Interaction happens as a consequence of an explicit request of the human to satisfy a goal or because the robot finds itself in a situation where it is useful if not mandatory. In both cases, the robot has a goal to satisfy. An important issue is the notion of engagement, a process in which the robot will have to establish, maintain and terminate a connection with a human partner. This covers goal establishment, selection of an incremental refinement of the task that is intended to be achieved, and execution control including monitoring, and even influencing, human task performance and his/her commitment to the goal. The human involvement may range from a direct participation to the task achievement, to a simple "acceptance" of robot activity in his/her close vicinity.

Our robot is controlled by a three layer architecture [1]. We present briefly its decisional layer. The proposed decisional framework consists of several entities, having each a specific role as illustrated by Figure 2. We describe how the robot is controlled through an analysis of the three main activities performed by the robot controller:

1) Situation assessment and context management

2) Goals and plans management

3) Action refinement, execution and monitoring

The next three sections describe the three robot controller activities and how they make use of a number of key compo- 


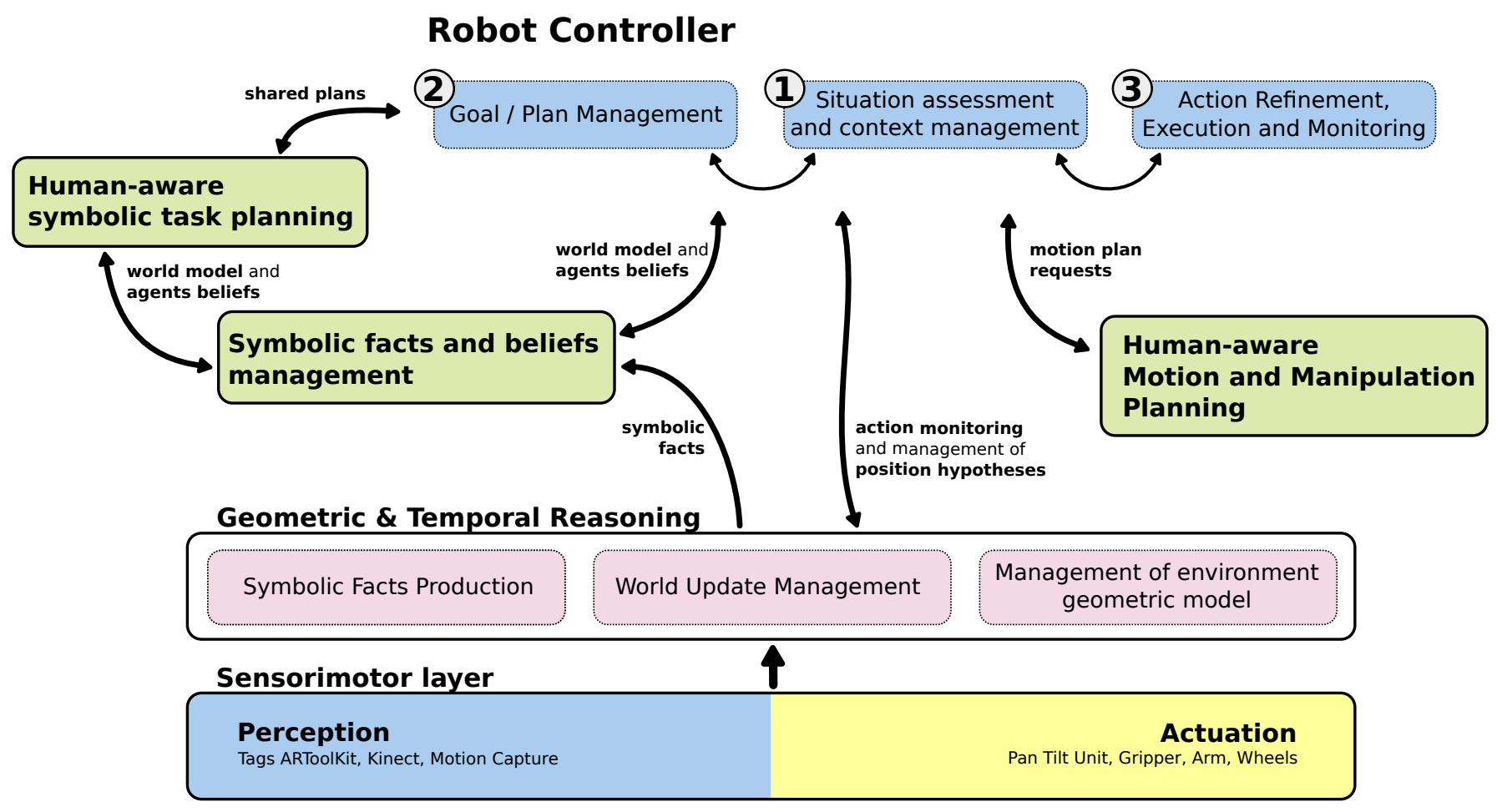

Figure 2. Architecture of the robot control system

nents in the architecture:

- SPARK: Spatial Reasoning and Knowledge module [12]

- ORO: a knowledge management module [3]

- HATP: a Human-Aware Task Planner [2]

- A set of Human aware motion, placement and manipulation planners [6], [8], [10]

Other decisional activities, such as situated dialog ( [4], [9], not presented here) have been developed that use the same set of components.

\section{Situation ASSESSMent AND CONTEXT MANAGEMENT}

This activity involves the geometric and temporal reasoning component, the symbolic facts and belief management component and the dedicated robot controller activity (Figure 2).

Geometric reasoning plays a central role in our architecture. It is performed by a component called SPARK (Spatial Reasoning and Knowledge [12]) in the current implementation. It is responsible for geometric information gathering and it embeds a number of decisional activities linked to abstraction (symbolic facts production) and inference based on geometric and temporal reasoning. SPARK maintains all geometric positions and configurations of agents, objects and furniture coming from perception and previous or a priori knowledge.

Reasoning about human perspective allows to compute facts such as: 〈GREY_TAPE isBehind HUMAN1), 〈GREY_TAPE isVisibleBy HUMAN1〉.

Monitoring human activity is crucial to maintain a coherent state of the world. Full human action and activity monitoring is a difficult task that requires knowledge and reasoning both on high level facts like goals, intentions and plans, as well as bottom-up data from agent and object motions. Simple temporal and geometric reasoning on human hand trajectories and potential objects placements can provide some useful clues for high level human monitoring processes.

The facts produced by the geometric and temporal reasoning component are stored in a central symbolic knowledge base, called ORO. Besides acting as a facts database, the ORO platform [3] exposes several functions: operations on knowledge statements relying on inference (through a continuous firstorder logic classification process), management of per-agent symbolic models, and also higher cognitive and human-robot interaction related functionalities like categorization of sets of concepts and natural language grounding [4].

\section{Goal and Plan Management}

In order to devise how a given goal can be accomplished, the robot has to elaborate a plan, i.e. a set of actions to be achieved by itself and its human partners. This is the role of HATP [2] (for Human Aware Task Planner). HATP is based on a Hierarchical Task Network (HTN) refinement which performs an iterative task decomposition into sub-tasks until reaching atomic actions [7].

One key feature is that HATP is able to produce plans for the robot actions as well as for the other participants (humans or robots).

The resulting plan, called "shared plan" is a set of actions that form a stream for each agent involved in the goal 
achievement. Depending on the context, some "shared plans" contain causal relations between agents.

It can be tuned by setting up different costs depending on the actions to apply and by taking into account a set of constraints called social rules. This tuning aims at adapting the robot behavior according to the desired level of cooperation of the robot [2].

Depending on the context and on the shared plan elaborated by HATP for a given goal, the robot controller decides to execute an action or to ask its human partner to do it. Actions feasibility by the human or the robot are regularly reconsidered based on the reachability / visibility computation mechanisms.

\section{An ILlustrative EXAMPLE}

Let us take a simple example to illustrate a full run of the system. We assume here that the robot (and the human) has been given the joint goal "CLEAN TABLE". For HATP, this means putting all tapes that are currently on the table in the trashbin. Depending on the state of the world and agent preferences, different plans are produced.

There is only one tape on the table and it is is reachable only by the robot while the trashbin is reachable only by the human.

Figure $\mathrm{V}$ illustrates the main processes occurring during a multi-step human-robot collaborative goal achievement. The plan produced is quite straightforward and is shown in the third row called "Goal and Plan". It consists in 4 successive actions involving the robot and the human. Robot grasps the tape and then places it on the table at a position where it is visible and reachable for the human. Human then is asked to pick the tape and to throw it in the trashbin. The first row, named "Cameras", shows several snapshots corresponding to various execution steps. Snapshot 1 corresponds to the initial situation. Snapshots 2, 3, 4 and 5 give the state after the successive achievement of the four actions in the plan. The second row, named "3D Model", shows the display of SPARK at the same instants. The fourth row, called "Robot Speech Acts", illustrates robot speech acts produced along the execution to inform the human partner about goal and plan creation and status and to verbalize the actions that the human is asked to execute. The fifth row illustrates robot knowledge on itself and on the objects. The sixth row illustrates the robot knowledge about the human state. The seventh row gives ongoing robot action with action preconditions and effects assessment as well as motion execution tasks. The eighth row gives ongoing human action with action preconditions and effects assessment and monitoring activity.

\section{ACKNOWLEDGMENT}

This work has been conducted within the EU SAPHARI project (http://www.saphari.eu/) funded by the E.C. Division FP7-IST under Contract ICT-287513.

We also would like to thank warmly a number of LAAS robotics team members and more particularly Severin Lemaignan, Matthieu Warnier, Julien Guitton, Emrak Akin Sisbot, Samir Alili, Xavier Broquere, Mokhtar Gharbi, Wuwei He,
Matthieu Herrb, Jim Mainprice, Raquel Ros, Daniel Sidobre, Thierry Siméon.

\section{REFERENCES}

[1] R. Alami, R. Chatila, S. Fleury, M. Ghallab, and F. Ingrand. An architecture for autonomy. International Journal of robotics Research, Special Issue on Integrated Architectures for Robot Control and Programming, 17(4), 1998.

[2] S. Alili, V. Montreuil, and R. Alami. HATP task planner for social behavior control in autonomous robotic systems for hri. In The 9th International Symposium on Distributed Autonomous Robotic Systems, 2008.

[3] S. Lemaignan, R. Ros, L. Mosenlechner, R. Alami, and M. Beetz. Oro, a knowledge management module for cognitive architectures in robotics. In Proceedings of the 2010 IEEE/RSJ International Conference on Intelligent Robots and Systems, 2010.

[4] S. Lemaignan, A. Sisbot, and R. Alami. Anchoring interaction through symbolic knowledge. In Proceedings of the 2011 Human-Robot Interaction Pioneers workshop, 2011.

[5] S. Lemaignan, Raquel Ros, E. Akin Sisbot, Rachid Alami, Michael Beetz Broqure, D. Sidobre, R. Alami Grounding the interaction: anchoring situated discourse in everyday human-robot interaction Acceptable Robot Motions International Journal of Social Robotics, Volume 2, Issue 3, pp 329-343, April 2012

[6] J. Mainprice, E.A. Sisbot, L. Jaillet, J. Cortes, R. Alami, and T. Simeon. Planning human-aware motions using a sampling-based costmap planner In IEEE International Conference on Robotics and Automation, 2011.

[7] D. Nau, T. C. Au, O. Ilghami, U. Kuter, J. W. Murdock, D. Wu, and F. Yaman. Shop2: An htn planning system. Journal of Artificial Intelligence Research, pages 379-404, 2003.

[8] A.K. Pandey and R. Alami. Mightability maps: A perceptual level decisional framework for co-operative and competitive human-robot interaction. In IEEE/RSJ International Conference on Intelligent Robots and Systems, 2010.

[9] R. Ros, S. Lemaignan, E.A. Sisbot, R. Alami, J. Steinwender, K. Hamann, and F. Warneken. Which one? grounding the referent based on efficient human-robot interaction. In 19th IEEE International Symposium in Robot and Human Interactive Communication, 2010.

[10] E. A. Sisbot, A. Clodic, R. Alami, and M. Ransan. Supervision and motion planning for a mobile manipulator interacting with humans. 2008

[11] E.A Sisbot, L.F. Marin-Urias, X. Broqure, D. Sidobre, R. Alami Synthesizing Robot Motions Adapted to Human Presence. A Planning and Control Framework for Safe and Socially Acceptable Robot Motions International Journal of Social Robotics Volume 2, Issue 3, pp 329-343, 2010

[12] E. A. Sisbot, R. Ros, and R. Alami. Situation assessment for humanrobot interaction. In 20th IEEE International Symposium in Robot and Human Interactive Communication, 2011.

[13] E. A. Sisbot, and Rachid Alami A Human-Aware Manipulation Planner IEEE Transactions on Robotics, ISSN : 1552-3098, Digital Object Identifier : 10.1109/TRO.2012.2196303, 2012. 


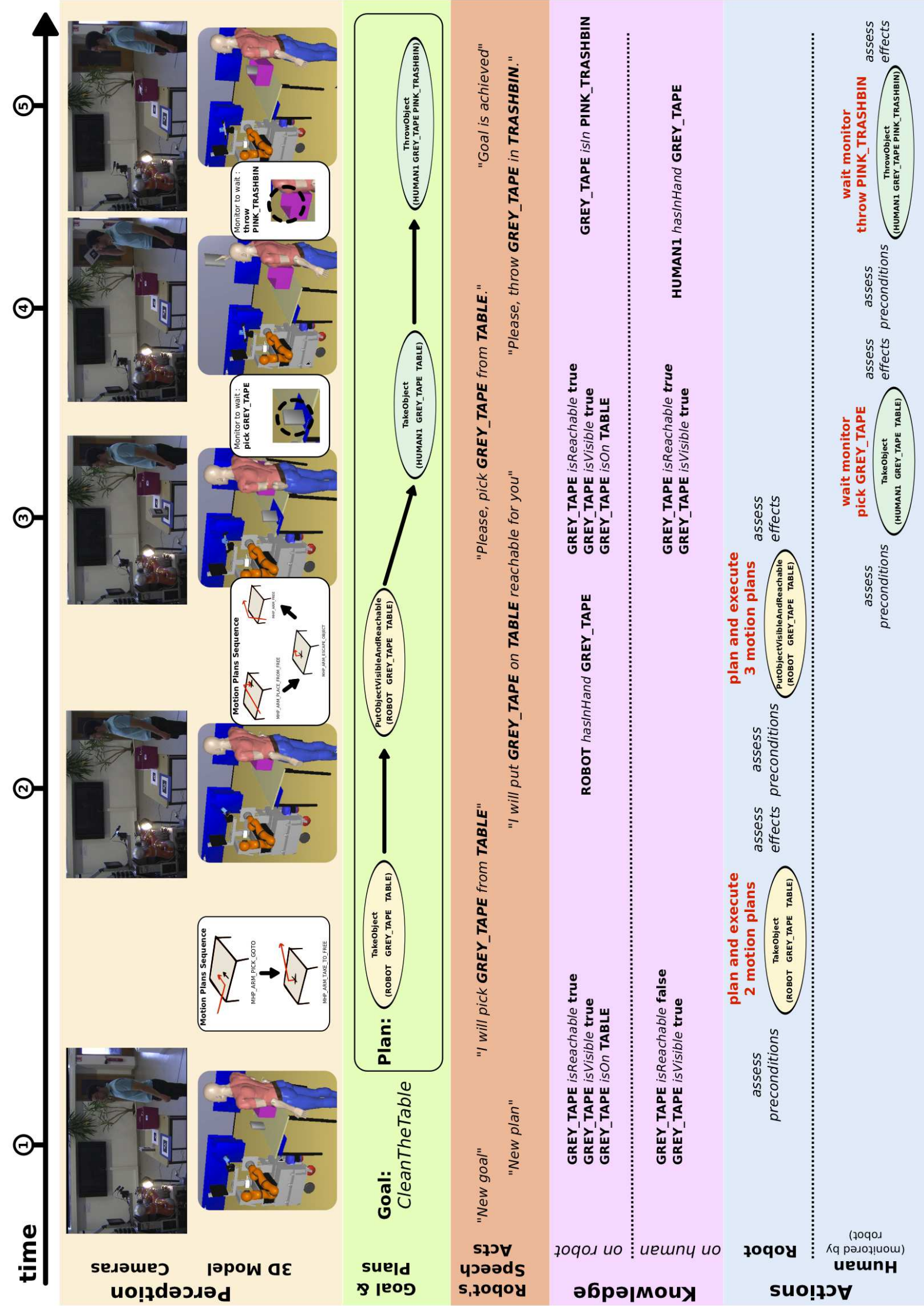

Figure 3. The main processes occurring during a multi-step human-robot collaborative goal achievement 